\title{
Filtering Strategies for TFC Selection Schemes in 3GPP W-CDMA Systems
}

\author{
Sheng-Tzong Cheng, Chun-Yen Wang, and Ing-Ray Chen, Member, IEEE
}

\begin{abstract}
The selection of a suitable transport format combination (TFC) according to the system load condition is one important issue of the radio resource management (RRM) in 3GPP W-CDMA systems. Different TFCs specify different ways of transmitting user data input from logical channels. It is observed that data transmission with a higher data rate will suffer a higher bit error rate (BER). This paper investigates the impact of BER on the selection of optimal TFC for transmitting the user data. Two filtering strategies are proposed to filter out infeasible TFCs so that the optimal TFC can be selected to achieve high performance. Moreover, an imbedded Markov chain is developed to evaluate the proposed strategies. From the simulation results, the feasibility and the effectiveness of the proposed strategies are demonstrated as well. We are suggesting that, for the design of an optimal TFC selection algorithm, the proposed TFC filtering strategy shall be taken into consideration.
\end{abstract}

Index Terms-3GPP, BER, QoS, MAC, RRM, SF, TFC, and W-CDMA.

\section{INTRODUCTION}

A $\mathrm{N}$ important feature of the future broadband wireless communications will be the ability to dynamically support various data rates and efficiently manage the radio resource. Radio resource management (RRM) plays a significant role in providing the desired quality of services in data delivery over the air interface. RRM is also responsible for efficient utilization of the radio resource to support various classes of traffic [14]. In order to utilize the air resource, newly developed 3GPP W-CDMA systems support variable bit rates for data communication on demand.

The radio interface protocol of $3 \mathrm{GPP}$ is decomposed into the following three layers: the physical layer (layer 1), the data link layer (layer 2) and the network layer (layer 3) [1]. Layer 2 is further split into four sub layers: Medium Access Control (MAC), Radio Link Control (RLC), Packet Data Convergence Protocol (PDCP) and Broadcast/Multicast Control (BMC). Among these layers, one of the crucial functions of the MAC protocol is the selection of appropriate transport format for each transport channel depending on instantaneous data rate of logical channels.

Manuscript received September 17, 2003; revised February 28, 2004 and March 3, 2005; accepted May 8, 2005. The associate editor coordinating the review of this paper and approving it for publication was A. Sheikh. This work was supported in part by the Institute of Information Industry, Taiwan, under the contract of 91-C-33.

S.-T. Cheng and C.-Y. Wang are with the Department of Computer Science and Information Engineering, National Cheng Kung University, Tainan 701, Taiwan (e-mail: stcheng@mail.ncku.edu.tw; eric@csie.ncku.edu.tw).

I.-R. Chen is with the Computer Science Department, Virginia Tech, Northern Virginia Center, 7054 Haycock Road, Falls Church, VA 22043 (email: irchen@cs.vt.edu).

Digital Object Identifier 10.1109/TWC.2006.05010.
Transport channels are responsible for exchanging data between MAC layer and physical layer. Data arriving from each logical channel must be mapped into a transport channel at MAC layer. And a set of combinations of transport formats of each transport channel is given by the radio resource control (RRC) layer for selection. This mechanism for MAC to select a suitable transport format combination (TFC) is known as the TFC selection scheme [7]. Note that data delivery using different transport format combination consumes various transmission power and results in different data rates and errorbit rates. As a result, the problem of selecting a suitable TFC according to the system load condition is one important issue of the RRM.

This paper studies the relationship between a TFC and its corresponding spreading factor (SF) and suggests that the bit error rate (BER) of a radio frame is a useful index to filter out some infeasible TFCs in a given TFCS. Actually, in WCDMA systems, power control is commonly used to keep the received Signal-to-Interference ratio (SIR) equal to a given target according to the received BER. However, in an errorprone wireless environment, we would show it later that it not enough for a limited power UE to only adopt the power control to keep the BER of the data transmission.

In this paper, we investigate the impact of the BER on the TFC selection algorithms. Two TFC filtering strategies, the throughput based and QoS based filtering strategies, are proposed to improve the performance of the TFC selection schemes for W-CDMA systems in the uplink. The proposed TFC filtering strategies can not only reduce the complexity of TFC selection algorithms but also enable the traditional TFC selection algorithms to dynamically adjust the transmission data bit rate according to the current traffic load and the quality of the transmission in the air.

More precisely, the throughput based filtering strategy attempts to filter out some TFCs with lower effective throughput and higher BER value so that the TFC selection mechanism is able to achieve the maximum throughput. In addition, the QoS based filtering strategy tries to satisfy the various QoS requirements so that data is transmitted without exceeding its maximum tolerable BER value (MTBER).

Note that the functionality among the TFC filtering strategies, TFC elimination, and TFC blocking [5][6] are different. All of these mechanisms are applied to determine the candidate TFCs for the TFC selection schemes. However, TFC elimination and TFC blocking schemes only remove those TFCs that require a transmission power higher than the maximum UE transmitter power. But the proposed TFC filtering strategies filter out those infeasible TFCs more stringently. 
Even though the transmission power for a given TFC can be supported by the UE, it would be beneficial to filter out the TFC if it would suffer an overhigh BER value.

In addition, an imbedded Markov chain has been developed to approximate the proposed TFC filtering strategies. This queueing model makes it convenient to estimate the average number of packets waiting in the buffers and the mean packet waiting time. Besides, simulations are also conducted to evaluate the performance of the proposed filtering strategies. Results reveal that, for the design of an optimal TFC selection algorithm, the TFC filtering strategies shall be taken into account.

The rest of this paper is organized as follows. In Section II, the general concepts about the TFCS are introduced. Related research is surveyed as well. In Section III, the relationship among the TFCS, the SF, and the BER are investigated. In Section IV, the proposed TFC filtering strategies are depicted. In Section V, an imbedded Markov chain is presented to approximate the proposed filtering strategies. In Section VI, experiments are conducted to evaluate the performance of the proposed filtering strategies. Finally, conclusion remarks are drawn in Section VII.

\section{BACKGROUND AND RELATED WORK}

In this section, we introduce definitions and terms generally used in describing the transport channels throughout this paper. In-depth treatments are available in the 3GPP technical specifications [2].

\section{A. TFCS Parameters}

The transport channel is responsible for exchanging data between MAC layer and physical layer. The transport channels process the traffic from logical channels and send it to the physical layer. A Transport Block (TB) is the basic data unit through a transport channel. And a number of TBs, which are exchanged between layer 1 and MAC layer at the same time, is called Transport Block Set (TB set). The number of bits in a TB and a TB set are defined as the transport block size (TB size) and the transport block set size (TB set size), respectively.

Furthermore, a transport channel always delivers a specific TB set to the physical layer in a fixed period of time when there is data to be transmitted. This period is defined as the Transmission Time Interval (TTI). It must be a multiple of the minimum interleaving period (e.g., $10 \mathrm{~ms}$ ).

The Transport Format describes the attributes for the delivery of a TB set during a TTI on a transport channel. The transport format consists of two parts, dynamic part and semistatic part. Attributes of the dynamic part include TB size and TB set size (i.e., the number of TBs exchanged during a TTI). On the other hand, attributes of the semi-static part include TTI, error protection schemes (e.g., turbo code, convolutional code, no coding), coding rate, static rate matching parameter, size of cyclic redundancy check (CRC), etc.

For each transport channel, there exists a list of transport formats. However, at a given point of time, not all combinations of transport formats of all transport channels are applicable to physical layer but only a subset. The Transport
TABLE I

A SiMPLE EXAMPLE FOR TFCS

\begin{tabular}{|l|c|c|c|c|}
\hline \multicolumn{2}{|c|}{ TFCS } & DCH1 (bits) & DCH2 (bits) & DCH3 (bits) \\
\hline \multirow{3}{*}{ TFCI } & TFC 1 & $\{20,20\}$ & $\{320,1280\}$ & $\{320,320\}$ \\
\cline { 2 - 5 } & TFC 2 & $\{20,40\}$ & $\{320,1280\}$ & $\{320,320\}$ \\
\cline { 2 - 5 } & TFC 3 & $\{20,160\}$ & $\{320,320\}$ & $\{320,320\}$ \\
\hline CRC & 16 bits & 16 bits & 16 bits \\
\hline Coding Scheme & Convolution code & Convolution code & Turbo code \\
\hline Coding Rate & rate $=1 / 3$ & rate $=1 / 3$ & rate $=1 / 3$ \\
\hline TTI & $10 \mathrm{~ms}$ & $10 \mathrm{~ms}$ & $40 \mathrm{~ms}$ \\
\hline
\end{tabular}

Format Combination (TFC) is defined as the currently valid combination of transport format of all transport channels.

\section{B. Transport Format Combination Set}

The set of TFCs is referred as the Transport Format Combination Set (TFCS). When mapping data onto physical layer, MAC selects an appropriate TFC within the TFCS for each transport channel depending on instantaneous data rate of logical channels. So the data bit rate can be changed very fast without any need for layer 3 signaling. As a result, the selection of TFCs can be seen as a dynamic part of the RRC [3]. This assures the flexible variable data rate scheme provided by layer 1 can be fully utilized.

An example of a TFCS is given in TABLE $I$ in which three possible TFCs are defined. Each TFC consists of transport format of each transport channel. In addition, the dynamic and semi-static parts of each transport format are shown as well. The bit numbers in the parenthesis (e.g., $\{320$ bits, 1280 bits $\}$ ) indicate TB size and TB set size respectively. And the TTIs for DCH1, DCH2 and DCH3 are $10 \mathrm{~ms}, 10 \mathrm{~ms}$ and 40 ms respectively.

\section{Related Work}

In literature, scheduling issues in the MAC layer of WCDMA networks are studied and analyzed [8]. Principles of TFC selection algorithms are proposed and studied. A load priority based algorithm suggests that the logical channel with the highest priority shall be selected. On the other hand, an algorithm with fairness mechanism takes into account the overloaded channels. It tried to insure whether at least one packet from each logical channel is sent at each period. Besides, a combined scheme that uses load priority but considers overloaded logical channels is also proposed. A simulator is developed to assess the impact of the algorithms on the overall system behavior.

In addition, work has been reported in [9] and [10] on evaluating several decentralized MAC strategies for the selection of a suitable uplink transmission rate among the set of the allowed values. Three strategies: delay-oriented, service credit, and maximum rate (MR) algorithms are proposed. The delayoriented strategy tries to guarantee a specific delay bound for each packet. The service credit strategy uses the difference between the obtained bit rate and the expected bit rate as an index to allocate the number of transport blocks for a connection. The maximum rate algorithm selects a TFC that allows the maximum bit rate for a connection. The impact 


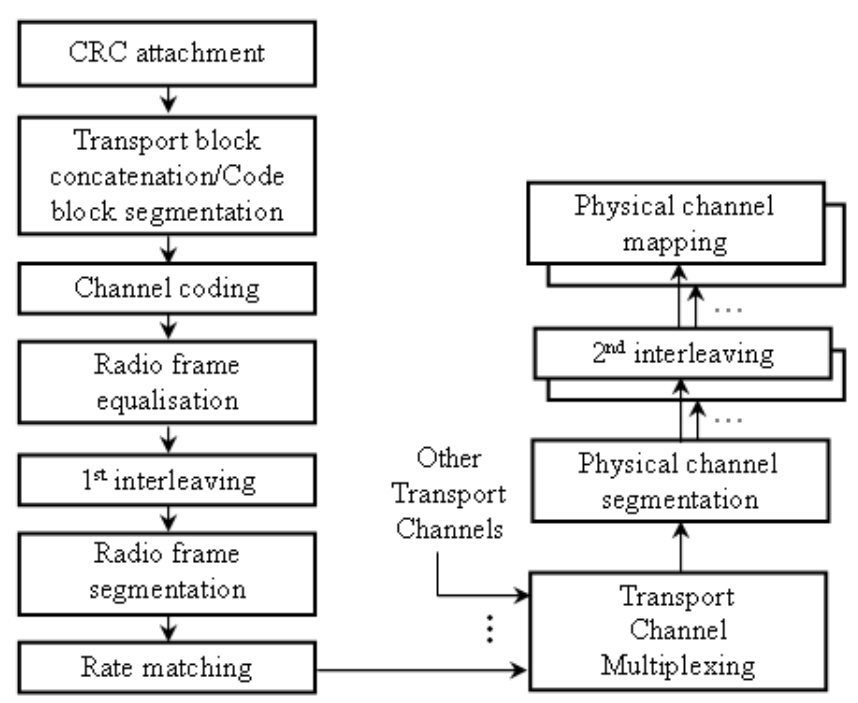

Fig. 1. Transport channel multiplexing structure in uplink.

of these three algorithms on admission control decisions is studied.

A packet scheduling strategy for the quality of service guarantee is developed in [11]. It checks the target quality consistently in the radio link with the priority level assigned to each user. The performance of the strategy is assessed by system level simulations.

We observe that, for different classes of services, the BER plays a key role in the quality of service of wireless networks [12]. In this paper, the relationship between the TFC, the SF, and the BER is established. Our work represents one of the few efforts on investigating the TFC impact on the BER and the throughput. The proposed TFC filtering strategy is able to dynamically adjust the candidate TFCs for the TFC selection algorithms according to the instantaneous transmission quality of the wireless link.

\section{The Relationship Between TFC, SF, AND BER}

Orthogonal variable spreading factor (OVSF) mechanism is exploited in W-CDMA systems to simultaneously support connections with various bandwidth requirements. The mechanism dynamically changes the data transmission rate and error rate by adjusting the spreading factor (SF). Once the desired data transmission rate is given, the corresponding SF can be indirectly determined. Accordingly, the BER of the transmission that uses a particular SF value is derived as well.

In this section, the relationship between the TFC, the SF, and the BER is established. It can be seen that each TFC has its corresponding SF value to transmit data while different SF values result in various BER values in transmission. Therefore, the relationship is essential to the development of an efficient TFC selection mechanism.

\section{A. Determination of Radio Frame Size}

The process of transport channel multiplexing in uplink is illustrated in Fig. 1 [4]. As shown, data in each transport channel is manipulated by the processes of CRC attachment, channel coding, code block segmentation, radio frame equalization,
TABLE II

TRANSPORT FORMAT V.S. SPREADING FACTOR

\begin{tabular}{|l|c|c|c|c|}
\hline \multicolumn{2}{|c|}{ TFCS } & DCH1 (bits) & DCH2 (bits) & SF \\
\hline \multirow{4}{*}{ TFCI } & TFC 0 & $\{160,160 \times 0\}$ & $\{320,320 \times 0\}$ & \\
\cline { 2 - 5 } & TFC 1 & $\{160,160 \times 1\}$ & $\{320,320 \times 1\}$ & $\mathrm{SF}_{1}=32$ \\
\cline { 2 - 5 } & TFC 2 & $\{160,160 \times 2\}$ & $\{320,320 \times 1\}$ & $\mathrm{SF}_{2}=16$ \\
\cline { 2 - 4 } & TFC 3 & $\{160,160 \times 2\}$ & $\{320,320 \times 2\}$ & $\mathrm{SF}_{3}=16$ \\
\cline { 2 - 4 } & TFC 4 & $\{160,160 \times 3\}$ & $\{320,320 \times 3\}$ & $\mathrm{SF}_{4}=8$ \\
\hline CRC & 16 bits & 16 bits & \multicolumn{1}{|c}{} \\
\hline Coding Scheme & Convolution code & Convolution code & \multicolumn{2}{|c}{} \\
\hline Coding Rate & rate $=1 / 3$ & rate $=1 / 3$ & \multicolumn{2}{|c}{$20 \mathrm{~ms}$} \\
\hline TTI & $10 \mathrm{~ms}$ & \multicolumn{2}{|c}{} \\
\hline
\end{tabular}

and rate matching. Each process adds a certain amount of data to the total volume. Notably, in the process of channel coding, data volume can be doubled or tripled depending on the coding rate.

Consider the case in which $N$ transport channels are contained in the $k$-th TFC of a given TFCS. Each transport channel $i$ has $n_{k, i}$ TBs to be transmitted in which each TB has the size of $b_{k, i}$ bits. After $N$ transport channels are multiplexed, the number of total data bits transmitted in a radio frame for the $k$-th TFC in bits, $S_{k}$, can be calculated by the following equation,

$$
\begin{gathered}
S_{k}=150 \times 2^{\left\lceil\log _{2} \hbar\right\rceil} \\
\hbar=\frac{1}{150 \times \min _{1 \leq i \leq N}\left\{R M_{i}\right\}} \times \sum_{i=1}^{N} \frac{n_{k, i} \times\left(b_{k, i}+C R C_{i}\right) \times R_{i} \times R M_{i}}{T T I_{i} / 10 m s},
\end{gathered}
$$

where $R M_{i}$ is the rate matching parameter of the transport channel $i$, and $1 / R_{i}$ denotes the coding rate of the transport channel $i$.

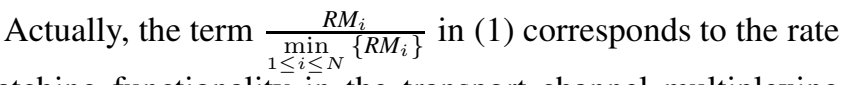
matching functionality in the transport channel multiplexing process (refer to Fig. 1). And the ceiling function is adopted to represent the functionality of the radio frame size equalization.

\section{B. The Relationship between Radio Frame Size and SF}

According to the $3 \mathrm{GPP}$ specifications, a radio frame has a fixed length of 38400 chips and shall be delivered in 10 $\mathrm{ms}$ (This corresponds to a chip rate of $3.84 \mathrm{M}$ chips per second). Therefore, the length of a TTI must be a multiple of $10 \mathrm{~ms}$. Namely, a TTI of $20 \mathrm{~ms}$ needs two radio frames for transmission. The value of SF indicates the number of chips for each data bit. For example, given the fact of 38400 chips for each radio frame, a radio frame with 600 bits needs 64 (= $38400 / 600$ ) chips for each bit. Consequently, the value of SF equals 64 . According to the 3GPP specifications, the feasible set for a radio frame size (in bits) is 9600, 4800, 2400, 1200, $600,300,150$. Therefore, the feasible set of SF values defined in OVSF is 4, 8, 16, 32, 64, 128, 256 accordingly.

Once the radio frame size is determined, the corresponding SF value for the $k$-th TFC can be computed by

$$
S F_{k}=\frac{38400}{S_{k}} \text {. }
$$

An example is given in TABLE II. Five TFCs are given in the example in which each TFC has two transport channels 
(i.e., DCH1 and DCH2). The TB sizes of DCH1 and DCH2 are 160 and 320 bits, respectively. The numbers of TBs in the five TFCs are $0,1,2$, and 3 . The corresponding SF value for each TFC is computed and shown in the last column.

\section{The Relationship between SF and BER}

SF has a significant impact on the BER in the uplink. The BER is a function of several factors, such as thermal noise, interference, modulation, and forward error-correction scheme being employed, etc. BER is also affected by the power control and signal strength. According to the Gaussian interference approximation [13], the channel BER when no forward error-correction scheme is employed can be evaluated by the following equation.

$$
B E R=\frac{1}{2} \operatorname{erfc}\left(\sqrt{\gamma_{b}}\right)=\frac{1}{2} \operatorname{erfc}\left(\sqrt{\frac{E_{b}}{N_{o}}}\right),
$$

where $\operatorname{erfc}()$ is the error function defined as $\operatorname{erfc}(x)=$ $\frac{2}{\sqrt{\pi}} \int_{x}^{\infty} e^{-t^{2}} d t$, and $E_{b} / N_{o}$ is the energy per bit to the noise density ratio [11]. It can be obtained by the following equation:

$$
\frac{E_{b}}{N_{o}}=\frac{E_{c} \times S F}{N_{o}}
$$

where $E_{c}$ is the chip power received by the Node B, and $N_{o}$ is the total interference that consists of the intra-cell interference $I_{o w n}$, the inter-cell interference $I_{\text {oth }}$, and background plus receiver noise $P_{B R}$. Namely, $N_{o}=I_{o w n}+I_{o t h}+P_{B R}$. From (3) and (4), it can be seen that more bits carried in a radio frame result in a smaller SF value and a larger BER value. Therefore, we establish the relationship between $S F_{k}, S_{k}$, and $B E R_{k}$ for the $k$-th TFC,

$$
B E R_{k}=\frac{1}{2} \operatorname{erfc}\left(\sqrt{\frac{E_{c} \cdot S F_{k}}{N_{o}}}\right)=\frac{1}{2} \operatorname{erfc}\left(\sqrt{\frac{E_{c}}{N_{o}} \cdot \frac{38400}{S_{k}}}\right) .
$$

\section{TFC Filtering StRATEGy}

It can be seen from (1) that a TFC with larger capacity transmits more bits in a radio frame (i.e., the TFC has a larger $S_{k}$ value). While according to (5), it can be seen that a TFC with a larger $S_{k}$ value will suffer a higher BER if the received $E_{c} / N_{o}$ is invariant. In W-CDMA systems, power control is commonly used to adjust the received $E_{c} / N_{o}$ value, so the BER value in data transmission can be kept. However, in an error-prone wireless environment, it is impossible for a UE with a limited power supply to keep the BER value under an acceptable level at all times. For this case, it is necessary to perform the TFC filtering strategy to filter out some infeasible TFCs with higher BER values based on the received $E_{c} / N_{o}$ value in the Node B.

In this section, two kinds of filtering strategies, the throughput based filtering strategy and the QoS based filtering strategy, are presented to improve the performance of the TFC selection algorithms. The purpose of the throughput based filtering strategy aims at achieving the maximum effective throughput whereas that of the QoS based filtering strategy tries to satisfy the various QoS requirements. The details of the proposed filtering strategies are described as follows.

\section{A. Throughput Based Filtering Strategy}

For a limited power UE, a TFC with larger capacity results in a larger radio frame size and a higher BER. Due to a higher BER, more data is lost in transmission. Consequently, the effective throughput of a TFC with larger capacity is not necessarily greater than that with smaller capacity.

Given a TFCS, the throughput based filtering strategy filters out infeasible TFCs and finds an optimal subset of TFCS such that the maximum effective throughput can be achieved based on the received $E_{c} / N_{o}$ value in the Node B.

Consider the $k$-th TFC with $N$ transport channels. Each transport channel $i$ has $n_{k, i}$ TBs to be transmitted in which each TB has the size of $b_{k, i}$ bits. Then the maximum effective throughput of the $k$-th TFC, $f_{k}$, can be computed by

$$
\begin{aligned}
f_{k} & =\sum_{i=1}^{N} \sum_{j=1}^{n_{k, i}} j \cdot b_{k, i}\left(\begin{array}{c}
n_{k, i} \\
j
\end{array}\right)\left[1-B L E R_{k, i}\right]^{j}\left[B L E R_{k, i}\right]^{n_{k, i}-j} \\
& =\sum_{i=1}^{N}\left[1-B L E R_{k, i}\right] n_{k, i} \times b_{k, i} .
\end{aligned}
$$

where $B L E R_{k, i}$ is the block error rate and $\left(\begin{array}{l}p \\ q\end{array}\right)$ denotes the binomial coefficient (i.e., $\left.\left(\begin{array}{l}p \\ q\end{array}\right)=\frac{p !}{q !(p-q) !}\right)$. Besides, in light of (5), when no coding scheme is considered, the $B L E R_{k, i}$ can be derived as

$$
B L E R_{k, i}=1-\left[1-\frac{1}{2} \operatorname{erfc}\left(\sqrt{\frac{E_{c}}{N_{o}} \cdot \frac{38400}{S_{k}}}\right)\right]^{b_{k, i}} .
$$

Hence, from (6) and (7), $f_{k}$ can be obtained by the following formula,

$$
f_{k}=\sum_{i=1}^{N}\left[1-\frac{1}{2} \operatorname{erfc}\left(\sqrt{\frac{E_{c}}{N_{o}} \cdot \frac{38400}{S_{k}}}\right)\right]^{b_{k, i}} \times n_{k, i} b_{k, i} .
$$

Consequently, it can be seen from (8) that $f_{k}$ depends on the $B E R_{k}$, the number of transmitted TBs, and the size of a TB in each transport channel (i.e., $b_{k, i}$ ). Accordingly, in order to achieve higher throughput, some TFCs with high BER values shall be filtered out.

It can be seen that, given a TFCS, there exists at least one TFC, say TFC $v$, with the maximum effective throughput value. The corresponding $B E R_{v}$ for the optimal TFC is used as the index to filter out any TFC with a higher BER value than $B E R_{v}$. Thus, the proposed throughput based filtering strategy can be stated as follows.

1. Compute the BER and the effective throughput for each TFC in a given TFCS.

2. Find the TFC with the maximum effective throughput and its corresponding BER value, say $b$.

3. Filter out any TFC whose BER value is bigger than $b$.

\section{B. QoS Based Filtering Strategy}

Consider a wireless network that supports several classes of multimedia traffic (e.g., voice, video, audio, e-mail, etc.). Each of these traffic classes represents specific QoS requirements, such as maximum tolerable BER value (MTBER) [12]. For example, voice data can typically tolerate BER up to $10^{-3}$, while data packets require BER below $10^{-9}$. Consequently, in 


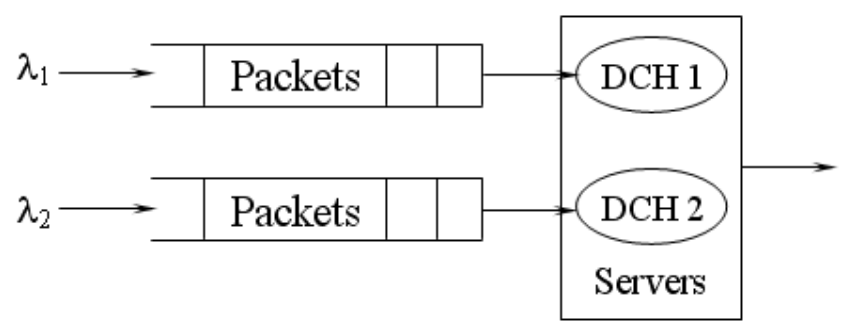

Fig. 2. The queueing model process.

order to guarantee the data transmitted without exceeding its MTBER value, the QoS based filtering strategy is proposed to filter out some infeasible TFCs.

Based on the current transmission quality in the air, the proposed QoS based filtering strategy filters out the TFCs that does not satisfy the most stringent MTBER specification among all the data that is transmitted at the same time.

Consider the $k$-th TFC in a given TFCS with $N$ transport channels. Each transport channel $i$ is responsible for transmitting the data of the traffic class $T_{i}$. Assume that each traffic class $T_{i}$ has a MTBER specification given by $M_{i}$. As a result, in order to satisfy the different MTBER requirements, the corresponding BER value, $B E R_{k}$, for the $k$-th TFC must be smaller than all the MTBER values of the data transmitted within it.

In the beginning, the QoS based filtering strategy computes the corresponding BER value for each TFC from (1) and (5). And then, for each TFC $k$, check whether it exists any transport channel $i$ such that $n_{k, i}>0$ and $B E R_{k}>M_{i}$. If there exists such a transport channel, then filter out the TFC. The proposed QoS based filtering strategy can be summarized as follows:

For $k=1$ to $K$ do

1. Compute the $B E R_{k}$ for the $k$-th TFC in a given TFCS.

2. Filter out the $k$-th TFC if there exists a $\operatorname{TrCH} i$ such that $n_{k, i}>0$ and $B E R_{k}>M_{i}$.

Since QoS based filtering strategy guarantees the most stringent MTBER requirements among all the data transmitted at the same time, it is wasteful to schedule simultaneous transmissions of various traffic classes. This observation is significant for developing an efficient TFC selection algorithm.

\section{Queueing Analysis}

In this section, an imbedded Markov chain [15] is employed to approximate the proposed algorithms. Without loss of generality, we consider the case that data is transmitted through two transport channels. As shown in Fig. 2, two servers, DCH1 and $\mathrm{DCH} 2$, are responsible for transmitting data in the buffer of logical channels LCH1 and LCH2, respectively. Assume that the sizes of packets arriving at $\mathrm{LCH} 1$ and $\mathrm{LCH} 2$ are equal to the TB sizes of DCH1 and DCH2. In addition, the packet arrival processes for $\mathrm{LCH} 1$ and $\mathrm{LCH} 2$ are Poisson processes with arrival rates $\lambda_{1}$ and $\lambda_{2}$. Besides, the service discipline for each server is governed by the TFC selection algorithm. The TFC selection algorithm is to select a suitable TFC for the servers in each TTI. Once a TFC is chosen, the server
TABLE III

The Optimal Subset OF TFCS

\begin{tabular}{|c|c|c|c|}
\hline TFCI & DCH1 (bits) & DCH2 (bits) & Probability \\
\hline 0 & $\left\{n_{1}, n_{1} \times C_{0}\right\}$ & $\left\{n_{2}, n_{2} \times D_{0}\right\}$ & $Q_{0}$ \\
\hline 1 & $\left\{n_{1}, n_{1} \times C_{1}\right\}$ & $\left\{n_{2}, n_{2} \times D_{1}\right\}$ & $Q_{1}$ \\
\hline$\cdots$ & $\cdots$ & $\cdots$ & $\cdots$ \\
\hline$R-1$ & $\left\{n_{1}, n_{1} \times C_{R-1}\right\}$ & $\left\{n_{2}, n_{2} \times D_{R-1}\right\}$ & $Q_{R-1}$ \\
\hline
\end{tabular}

capacities (i.e., the transmission data rates) can be determined as well. Therefore, the service pattern can be modeled as the bulk service with deterministic service time (i.e., TTI). That is, the entire system is modeled as a $M / D^{[Y]} / 2 / \infty / T F C$ selection algorithm queueing system.

After filtering out some unsuitable TFCs via the proposed TFC filtering strategy, the feasible TFC subset can be obtained. As shown in TABLE III, the TB sizes of DCH1 and DCH2 are $n_{1}$ and $n_{2}$, respectively. And $C_{r}$ and $D_{r}$ represent the numbers of TBs in the $r$-th TFC. Once the TFC selection algorithm chooses the $r$-th TFC to transmit data, the servers are able to serve at most $C_{r}$ packets in the buffer of LCH1 and $D_{r}$ packets in the buffer of LCH2 within a TTI. In other words, the transmitted data sizes of these two servers within a TTI are not independent but related to the TFC formats.

Besides that, the probability (i.e., $Q_{r}$ ) that any of the $R$ TFC is selected by the TFC selection algorithm is also given. The probability function $Q_{r}$ is determined by the packet arrival rate and the TFC selection algorithm. For example, consider a TFC selection algorithm that always chooses the TFC from a given TFCS randomly. Thus, the probability function $Q_{r}$ shall be defined as $Q_{r}=1 / R$ for $r=0,1, \cdots, R-1$. On the other hand, consider another TFC selection algorithm, the maximum rate algorithm $(M R)$, proposed by Pérez-Romero, Sallent, and Agusté [9]. The MR algorithm always selects the TFC with the highest transmission bit rate according to the amount of bits to be transmitted. In other words, the probability function $Q_{r}$, for the MR algorithm, shall be defined as $Q_{v}=1$ and $Q_{r}=0$ for any other $r \neq v$, where the $v$-th TFC is the TFC with the minimum capacity such that its data transmission rate is higher than the data arrival rate.

Let the stochastic process $U:[0,+\infty) \rightarrow \mathbf{N} \times \mathbf{N}: U(t)=$ $(m, n)$ denote that there are $m$ packets in the buffer of LCH1 and $n$ packets in the buffer of LCH2 at time $t$. Consequently, it is clear that $U(0)=(0,0)$, because these two buffers are empty initially. To simplify the calculation, we take a snapshot of the system at time instants immediately before the transport channels transmit data. Let $t_{\tau}=\tau \times T T I-\epsilon$ for $\epsilon>0$ and $\epsilon \rightarrow 0$. Let $X_{\tau}=U\left(t_{\tau}\right)$ for $\tau=1,2, \ldots$. And then the stochastic process $\left\{X_{\tau}, \tau=1,2, \ldots\right\}$ forms the imbedded Markov chain of the continuous-parameter stochastic process $\{U(t), t \geq 0\}$.

Let $\pi(m, n)$ denote the steady state probability that there are $m$ packets in the buffer of LCH1 and $n$ packets in the buffer of LCH2. That is to say, $\pi(m, n)=\lim _{\tau \rightarrow \infty} \operatorname{Pr}\left\{X_{\tau}=\right.$ $(m, n)\}$. Let $\phi(m)$ be the steady state probability that there are $m$ packets in the buffer of LCH1, and $\psi(n)$ be the steady state probability that there are $n$ packets in the buffer of LCH2. Clearly, $\pi(m, n)=\phi(m) \psi(n)$. 
Let $a_{m}(t)$ be the probability of $m$ packets arriving at LCH1 in a time interval of length $t$. Therefore,

$$
a_{m}(t)=\left\{\begin{array}{cl}
\frac{\left(\lambda_{1} t\right)^{m}}{m !} e^{-\lambda_{1} t} & \text { for } m=0,1,2, \ldots \\
0 & \text { otherwise }
\end{array}\right.
$$

Similarly, $b_{n}(t)$, the probability of $n$ packets arriving at LCH2 in $t$, can also be obtained from the following equation

$$
b_{n}(t)=\left\{\begin{array}{cl}
\frac{\left(\lambda_{2} t\right)^{n}}{n !} e^{-\lambda_{2} t} & \text { for } n=0,1,2, \ldots \\
0 & \text { otherwise }
\end{array} .\right.
$$

Let $a_{m}$ and $b_{n}$ represent the abbreviation of $a_{m}(T T I)$ and $b_{n}(T T I)$, respectively. Hence, $a_{m}=0=b_{m}$ for any $m<0$ and $\sum_{m=0}^{\infty} a_{m}=1=\sum_{n=0}^{\infty} b_{n}$. Depending on the properties of $a_{m}$ and $b_{n}$, the state transition probability, $p_{(i, j) \rightarrow(m, n)}$, from state $(i, j)$ to state $(m, n)$ can be obtained by the following equation

$$
\begin{aligned}
p_{(i, j)} & \rightarrow(m, n) \\
& =\operatorname{Pr}\left\{X_{n+1}=(m, n) \mid X_{n}=(i, j)\right\} \\
& =\sum_{r=0}^{R-1} Q_{r} a_{m-\max \left\{i-C_{r}, 0\right\}} b_{n-\max \left\{j-D_{r}, 0\right\}} .
\end{aligned}
$$

Hence, the global balance equation is found as

$$
\pi(m, n)=\sum_{i=0}^{\infty} \sum_{j=0}^{\infty} \pi(i, j) \times p_{(i, j) \longrightarrow(m, n)} .
$$

Substituting (11) in (12), we obtain the steady-state equation

$$
\pi(m, n)=\sum_{i=0}^{\infty} \sum_{j=0}^{\infty} \pi(i, j) \sum_{r=0}^{R-1} Q_{r} a_{m-\max \left\{i-C_{r}, 0\right\}} \times
$$

Let $A(y)=\sum_{m=0}^{\infty} a_{m} y^{m}$ and $B(z)=\sum_{n=0}^{\infty} b_{n} z^{n}$, and define the generating functions $\Phi(y), \Psi(z)$ of the steady state probabilities $\phi(m)$ and $\psi(n)$ as $\Phi(y)=\sum_{m=0}^{\infty} \phi(m) y^{m}$ and $\Psi(z)=\sum_{n=0}^{\infty} \psi(n) z^{n}$ for $|y| \leq 1$ and $|z| \leq 1$. The detailed derivations of the generating functions $\Phi(y)$ and $\Psi(z)$ are demonstrated in the Appendix. We only show the results as follows.

$$
\begin{gathered}
\Phi(y)=\frac{A(y) \sum_{r=0}^{R-1} Q_{r} \sum_{i=0}^{C_{r}-1} \phi(i)\left(y^{C}-y^{C+i-C_{r}}\right)}{y^{C}-A(y) \sum_{r=0}^{R-1} Q_{r} y^{C-C_{r}}}, \\
\Psi(z)=\frac{B(z) \sum_{r=0}^{R-1} Q_{r} \sum_{j=0}^{D_{r}-1} \psi(j)\left(z^{D}-z^{D+j-D_{r}}\right)}{z^{D}-B(z) \sum_{r=0}^{R-1} Q_{r} z^{D-D_{r}}} .
\end{gathered}
$$

where $C=\max _{0 \leq r \leq R-1}\left\{C_{r}\right\}$ and $D=\max _{0 \leq r \leq R-1}\left\{D_{r}\right\}$.

In order to get rid of the unknown probabilities $\phi(i)$ and $\psi(j)$ in the numerator of (14), we make use of the following theorem.

Theorem: If $h(z)$ is analytic and nonzero and $|h(z)|<1$ for $|z|<1$, then $h(z)-z^{n}$ has $n$ zeros in $|z| \leq 1$ [16].

Based on this theorem, it can be verified that the denominator of (14) has $C$ roots in $|y| \leq 1$ as long as the inequality $\left|A(y) \sum_{r=0}^{R-1} Q_{r} y^{C-C_{r}}\right|<1$ for $|y|<1$ is established. Due to

$$
\begin{aligned}
& \left|A(y) \sum_{r=0}^{R-1} Q_{r} y^{C-C_{r}}\right|=\left|\sum_{m=0}^{\infty} a_{m} y^{m}\right|\left|\sum_{r=0}^{R-1} Q_{r} y^{C-C_{r}}\right| \\
& \leq \sum_{m=0}^{\infty} a_{m}|y|^{m} \sum_{r=0}^{R-1} Q_{r}|y|^{C-C_{r}}<\sum_{m=0}^{\infty} a_{m} \sum_{r=0}^{R-1} Q_{r}=1,
\end{aligned}
$$

we know the polynomial $y^{C}-A(y) \sum_{r=0}^{R-1} Q_{r} y^{C-C_{r}}=0$ has $C$ roots in $|y| \leq 1$.

Since $\Phi(y)$ is analytic and bounded within the unit circle, all the zeros of the denominator within and on the unit circle must also make the numerator vanish. It has been confirmed that there are $C$ - 1 roots of the denominator in $|y| \leq 1$, while the $C$-th root is clearly $y=1$. Therefore, the numerator should be written as

$$
A(y) \times H \times(y-1) \times\left(y-\alpha_{1}\right) \times \cdots \times\left(y-\alpha_{C-1}\right),
$$

where $1, \alpha_{1}, \alpha_{2}, \ldots, \alpha_{C-1}$ are the coincident roots of the denominator and numerator.

By substituting (15) in (14), the generating function $\Phi(y)$ can be obtained by

$$
\Phi(y)=\frac{A(y) H(y-1)\left(y-\alpha_{1}\right) \cdots\left(y-\alpha_{C-1}\right)}{y^{C}-A(y) \sum_{r=0}^{R-1} Q_{r} y^{C-C_{r}}} .
$$

Since $\Phi(1)=1=A(1)$, we can use L'Hôpital's rule to get $H$

$$
\begin{gathered}
\Phi(1)=1=\lim _{y \rightarrow 1} \Phi(y)=\frac{H\left(1-\alpha_{1}\right) \cdots\left(1-\alpha_{C-1}\right)}{\sum_{r=0}^{R-1} C_{r} Q_{r}-A^{\prime}(1)} \\
\Rightarrow H=\frac{\sum_{r=0}^{R-1} C_{r} Q_{r}-\lambda_{1} \cdot T T I}{\left(1-\alpha_{1}\right) \cdots\left(1-\alpha_{C-1}\right)} .
\end{gathered}
$$

Therefore, the generating function $\Phi(y)$ can be computed by

$$
\begin{aligned}
\Phi(y)= & \frac{\sum_{r=0}^{R-1} C_{r} Q_{r}-\lambda_{1} \cdot T T I}{\left(1-\alpha_{1}\right) \cdots\left(1-\alpha_{C-1}\right)} \times \\
& \frac{A(y)(y-1)\left(y-\alpha_{1}\right) \cdots\left(y-\alpha_{C-1}\right)}{y^{C}-A(y) \sum_{r=0}^{R-1} Q_{r} y^{C-C_{r}}} .
\end{aligned}
$$

Then the steady state probability $\phi(m)$ can be computed by finding the coefficients of $y^{m}$ in the generating function $\Phi(y)$. And the average number of packets in the buffer of LCH1 in the steady state, $L_{1}$, can also be determined by

$$
\begin{array}{r}
L_{1}=\frac{\sum_{r=0}^{R-1} C_{r} Q_{r}+\sum_{r=0}^{R-1} C_{r}^{2} Q_{r}-\left(\lambda_{1} \cdot T T I\right)^{2}}{2\left(\sum_{r=0}^{R-1} C_{r} Q_{r}-\lambda_{1} T T I\right)} \\
-C+\sum_{i=1}^{C-1} \frac{1}{1-\alpha_{i}} .
\end{array}
$$

Finally, by applying the Little's formula, the mean waiting time in the buffer of LCH1, $W_{1}$, can also be calculated by

$$
\begin{array}{r}
W_{1}=\frac{1}{\lambda_{1}} \cdot\left[\frac{\sum_{r=0}^{R-1} C_{r} Q_{r}+\sum_{r=0}^{R-1} C_{r}^{2} Q_{r}-\left(\lambda_{1} \cdot T T I\right)^{2}}{2\left(\sum_{r=0}^{R-1} C_{r} Q_{r}-\lambda_{1} T T I\right)}\right. \\
\left.-C+\sum_{i=1}^{C-1} \frac{1}{1-\alpha_{i}}\right] .
\end{array}
$$

Likewise, the steady state probability $\psi(n)$, the average number of packets $L_{2}$ and the mean waiting time $W_{2}$ in the buffer of LCH2 in the steady state can also be obtained in the same form.

It is significant for the design of RRC to exploit the information of the average number of packets waiting in the buffer and the mean packet waiting time, especially in radio bearer control. The radio bearer control is used to reconfigure the transport and physical channels and also triggers transport 
TABLE IV

TRANSPORT FORMAT COMBINATION SET

\begin{tabular}{|c|c|c||c|c|c|}
\hline TFCI & DCH 1 & DCH 2 & TFCI & DCH 1 & DCH 2 \\
\hline 0 & $\{320,0\}$ & $\{640,0\}$ & 8 & $\{320,320\}$ & $\{640,1280\}$ \\
\hline 1 & $\{320,320\}$ & $\{640,0\}$ & 9 & $\{320,960\}$ & $\{640,640\}$ \\
\hline 2 & $\{320,640\}$ & $\{640,0\}$ & 10 & $\{320,0\}$ & $\{640,1920\}$ \\
\hline 3 & $\{320,0\}$ & $\{640,640\}$ & 11 & $\{320,640\}$ & $\{640,1280\}$ \\
\hline 4 & $\{320,960\}$ & $\{640,0\}$ & 12 & $\{320,320\}$ & $\{640,1920\}$ \\
\hline 5 & $\{320,320\}$ & $\{640,640\}$ & 13 & $\{320,960\}$ & $\{640,1280\}$ \\
\hline 6 & $\{320,0\}$ & $\{640,1280\}$ & 14 & $\{320,640\}$ & $\{640,1920\}$ \\
\hline 7 & $\{320,640\}$ & $\{640,640\}$ & 15 & $\{320,960\}$ & $\{640,1920\}$ \\
\hline
\end{tabular}

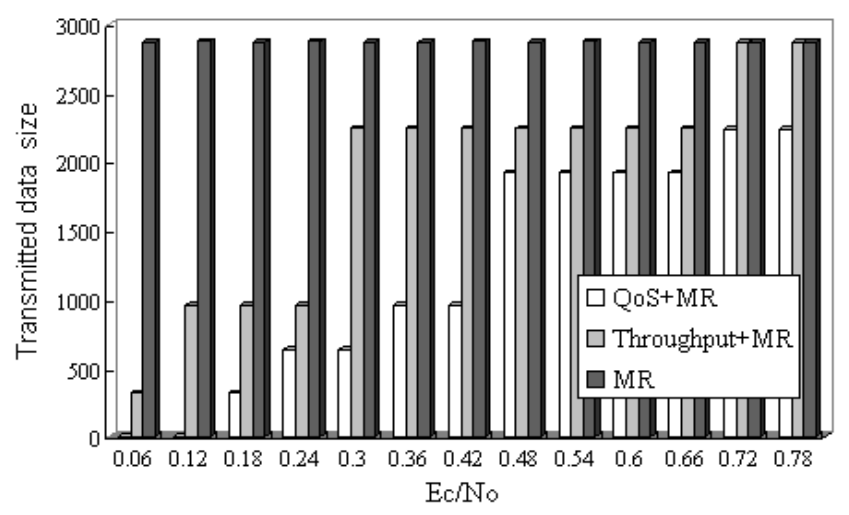

Fig. 3. Average transmitted data size in a TTI in various $E_{c} / N_{o}$ values.

channel type switching. In general, the radio bearer control is triggered by the amount of data waiting for transmission in the buffer. Consequently, by using (19) and (20), the RRC can not only configure the transport and physical channel more efficiently, but also reduce the frequency of radio bearer reconfiguration to achieve better effectiveness and utilization.

\section{Vi. Performance Evaluation}

In order to investigate the significance of the TFC filtering strategy, simulations have been carried out. The simulator and data generator are coded in $\mathrm{C}++$ programming language with the parameter settings referred to [3]. The Gaussian interference hypothesis is adopted to simulate the BER model in wireless transmission. In addition, we consider that the Node B would feedback the received $E_{c} / N_{o}$ value to the transmitters via the downlink control channels, so the UEs are aware of the current transmission quality in the air.

The simulation model assumes that two radio bearers (RBs), RB1 and RB2, within a UE are considered for supporting two kinds of services with MTBER values $10^{-6}$ and $10^{-4}$, respectively. The data arriving for transmitted by DCH1 and DCH2 are generated by uniform distribution with the parameters 900 1000 (bits/TTI) and 1800 1900 (bits/TTI), respectively. Possible TFCs (before performing the TFC filtering strategies) are detailed in TABLE IV. Suppose that no code scheme is employed, rate matching parameter is equal to 1 , and the TTI of each transport channel equals to $10 \mathrm{~ms}$. Besides that, the TFC selection algorithm, maximum rate algorithm (MR), is adopted here to demonstrate the effectiveness of the proposed TFC filtering strategies.
The MR selection algorithm selects the TFC with the highest transmission bit rate according to the amount of bits $L_{b}$ to be transmitted. Thus, the number of transport blocks to be transmitted would be

$$
\text { numTB }=\min \left(\text { TBmax }\left\lceil\frac{L_{b}}{\text { TBsize }}\right\rceil\right) .
$$

where TBmax is the maximum number of TBs allowed in a TTI, and TBsize is the number of bits in a TB for the considered radio access bearer (RAB). In order to realize how the filtering strategy operates, the performance of three different methods is investigated. One is the MR with the throughput based filtering strategy (Throughput+MR), another is the MR with the QoS based filtering strategy (QoS+MR), and the other is the original MR without any filtering strategy. The comparisons among these methods are presented as follows.

The results shown in Fig. 3 to Fig. 7 are obtained from the repeated simulation on various parameters over 1000 runs (i.e., 1000 TTIs). Each point shown in the curve represents the average value of the results over 1000 runs.

Fig. 3 shows the average transmitted data size per TTI in various transmission quality for different strategies. The transmitted data size means the sum of the transmitted data size in DCH1 and DCH2 based on the different mechanisms. It can be observed in Fig. 3 that the original MR constantly considers the whole TFCS, so it always transmits data with highest data rate. However, the proposed filtering strategies are able to dynamically select the optimum subset of TFCS based on the quality of transmission. In other words, the proposed filtering strategies enable the traditional TFC selection algorithms to dynamically adapt the data transmission rate according to the current transmission quality in the air.

In order to verify the issue that higher data rate suffers higher error bit rate, Fig. 4 presents the average number of error bits in a TTI for different strategies. To simulate the BER model in wireless transmission, the Gaussian interference hypothesis is also adopted. It can be seen that the average number of error bits for the original MR is much larger than that with the filtering strategy. That is because the original MR always selects the TFC with the highest bit rate no matter how the quality of transmission in the air is. Nevertheless, the TFC filtering strategies filter out infeasible TFCs, so the amount of the average error bits is much smaller than that of the original MR. Note that there are some discontinuous points in the curve for the MR with filtering strategies. That is because these methods select a different TFC from the former one due to the change of $E_{c} / N_{o}$. As a result, a different number of error bits are obtained. As the value of $E_{c} / N_{o}$ increases (i.e., the air link is more reliable), the number of error bits decreases until another discontinuous point occurs.

In addition, Fig. 5 presents the comparison of the average effective throughput per TTI in various $E_{c} / N_{o}$ for different methods. It can be seen that the MR with the throughput based filtering strategy achieves a higher effective throughput than the other methods do. For example, when $E_{c} / N_{o}=0.5$, the average effective throughput of the throughput based filtering strategy achieves 2203.52 bits in a TTI and the QoS based filtering strategy performs 1879.34 bits while the original MR is only 902.123 bits. 


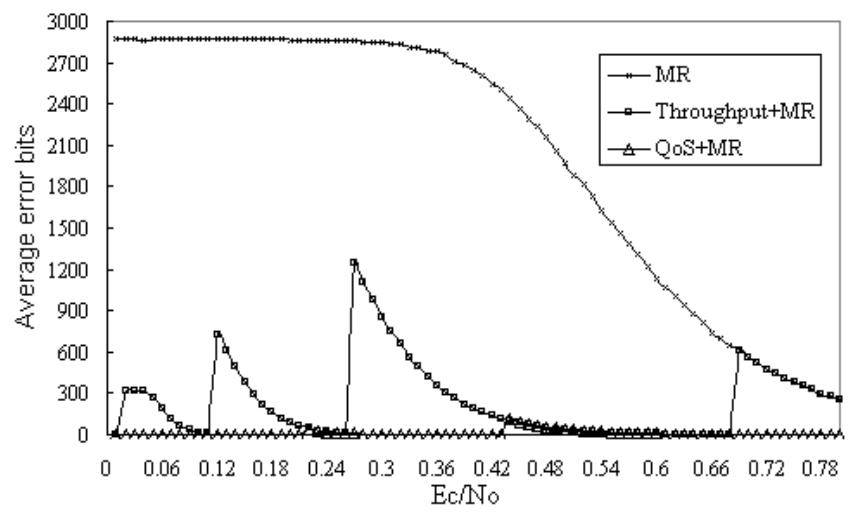

Fig. 4. Comparison of the average error bits in various $E_{c} / N_{o}$.

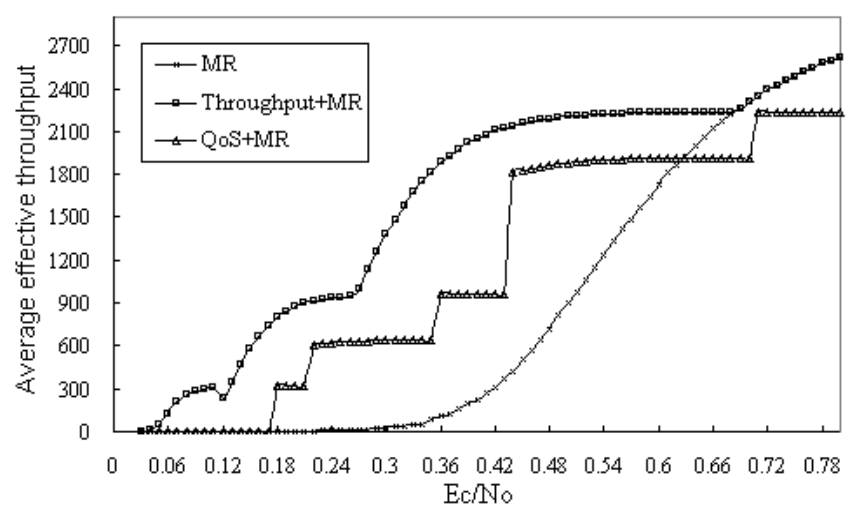

Fig. 5. Comparison of the average effective throughput in various $E_{c} / N_{o}$.

Fig. 6 and Fig. 7 depict the average BER value for DCH1 and $\mathrm{DCH} 2$, respectively. DCH1 is responsible for transmitting the data of the RB1 whose MTBER is equal to $10^{-6}$. It can be observed in Fig. 6 that only the QoS based filtering strategy holds the BER values below the bound of $10^{-6}$ regardless of the transmission quality. Similarly, DCH2 is responsible for transmitting the data of RB2 whose MTBER equals $10^{-4}$. As shown in Fig. 7, among these three methods, only the QoS based filtering strategy can keep the BER value below the bound of $10^{-4}$.

Finally, the analytic results from the queueing model are compared with those from the simulation. Fig. 8 and Fig. 9 present the average number of packets waiting in the queue of LCH1 and LCH2, respectively. Each point shown in the curve of approximation is obtained by calculating (19) while each point shown in the curve of simulation results represents the average value of the results on various parameters over 6000 runs. In the simulation model, we consider that the packet arrival process for $\mathrm{LCH} 1$ and $\mathrm{LCH} 2$ are both Poisson process with arrival rate varying from 0.8 to 1.9 (packets/TTI). Moreover, the number of bits per packet of LCH1 is 320 bits, and the number of bits per packet of $\mathrm{LCH} 2$ is 640 bits. Consider the case when $E_{c} / N_{o}$ is set to 0.7. The TFCS parameters shown in TABLE IV are also adopted. In addition, the MR selection algorithm with the throughput based filtering strategy is used to transmit data. As shown in Fig. 8 and Fig. 9 , it can be observed that the curves of our approximation are



Fig. 6. Comparison of the BER for DCH1 in various $E_{c} / N_{o}$.

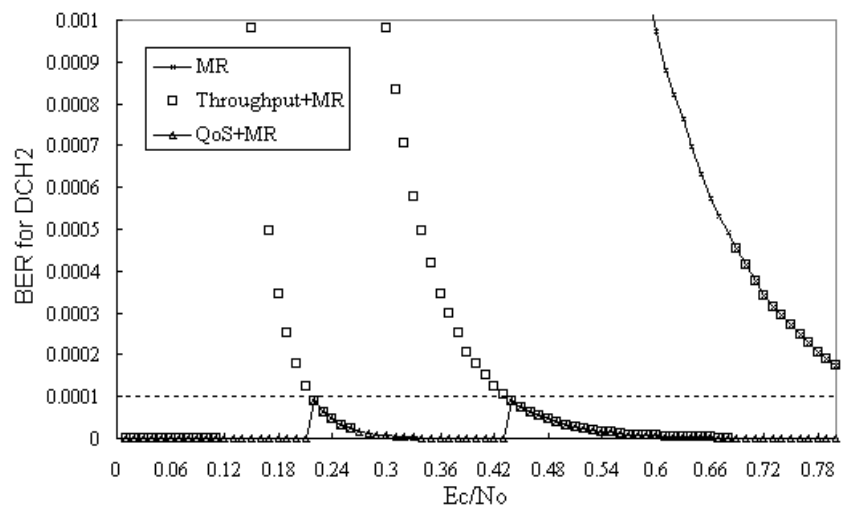

Fig. 7. Comparison of the BER for DCH2 in various $E_{c} / N_{o}$.

very close to that of numerical results.

In sum, the proposed filtering strategies enable the traditional TFC selection algorithms to dynamically adjust the data rate according to the transmission quality in the air. Furthermore, from the simulation results, it has been shown that the throughput based filtering strategy achieves the maximum effective throughput and the QoS based filtering strategy always keeps the data transmitted below the corresponding MTBER value. At last, the accuracy of the proposed approximation model is also verified by numerical results.

\section{CONCLUSION}

In $3 \mathrm{G}$ communication systems, new and efficient radio resource management algorithms for the load control and interference management are required to maximize the system throughput. TFC selection algorithm is an important part of the radio resource management for data transmission. Focusing on the influence of the BER, this paper proposes two filtering strategies, the throughput based and QoS based filtering strategies, to filter out infeasible TFCs for the TFC selection algorithms. From the simulation results, it can be seen that the throughput based filtering strategy always achieves the maximum effective throughput and the QoS based filtering strategy always keeps the data transmitted below its MTBER value. In addition, the proposed filtering strategies enable the traditional TFC selection algorithms to dynamically adjust the transmission data bit rate according to the current quality of the transmission in the air. Furthermore, an imbedded Markov 


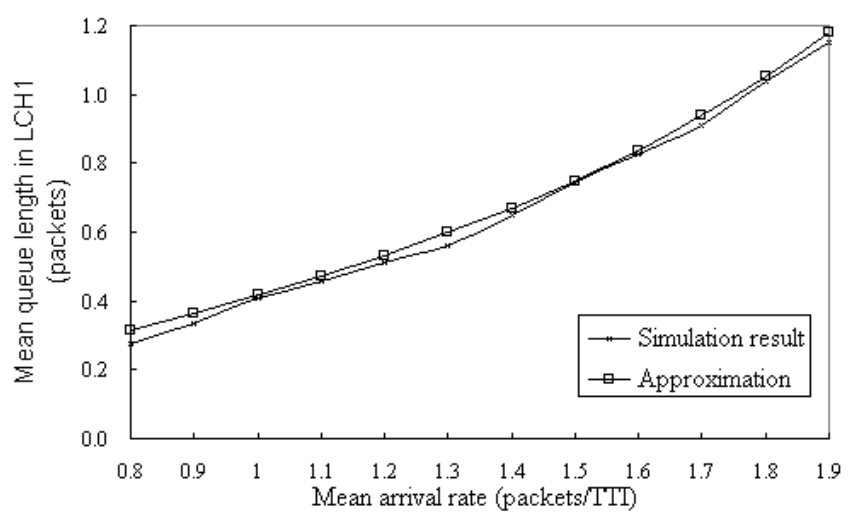

Fig. 8. Mean number of packets in the buffer of LCH1 for $E_{c} / N_{o}=0.7$.

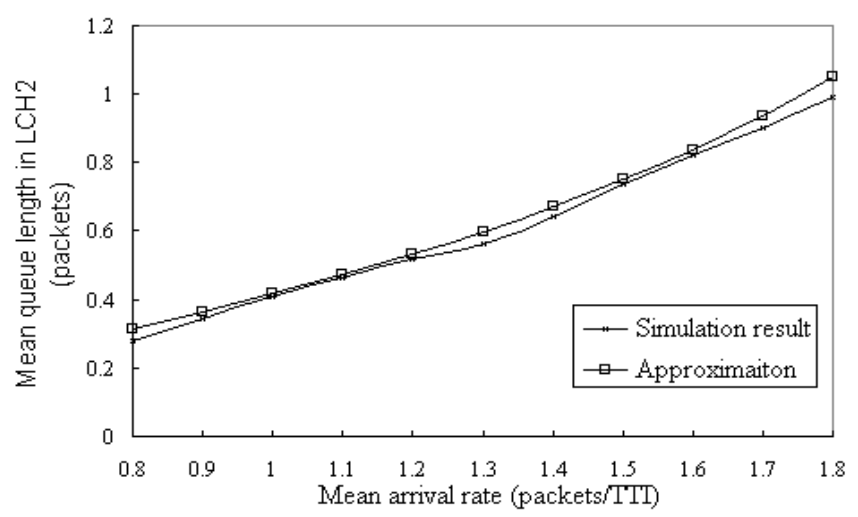

Fig. 9. Mean number of packets in the buffer of $\mathrm{LCH} 2$ for $E_{c} / N_{o}=0.7$.

chain is developed to evaluate the proposed filtering strategies. Based on these advantages, it can be seen that the proposed filtering strategies are scalable and can be applied to increase the efficiency of the data transmission in WCDMA uplink.

\section{APPENDIX}

In the appendix, we present the derivation of the generating functions $\Phi(y)$ and $\Psi(z)$ that are defined in Section V using the same notations as follows.

By expanding (13), we can obtain the steady-state equation

$$
\begin{gathered}
\pi(m, n)=\sum_{r=0}^{R-1} Q_{r} \sum_{i=0}^{C_{r}-1} \sum_{j=0}^{D_{r}-1} \pi(i, j) a_{m} b_{n}+ \\
\sum_{r=0}^{R-1} Q_{r} \sum_{i=0}^{C_{r}-1} \sum_{j=D_{r}}^{n+D_{r}} \pi(i, j) a_{m} b_{n-\left(j-D_{r}\right)}+ \\
\sum_{r=0}^{R-1} Q_{r} \sum_{i=C_{r}}^{m+C_{r}} \sum_{j=0}^{D_{r}-1} \pi(i, j) a_{m-\left(i-C_{r}\right)} b_{n}+ \\
\sum_{r=0}^{R-1} Q_{r} \sum_{i=C_{r}}^{m+C_{r}} \sum_{j=D_{r}}^{n+D_{r}} \pi(i, j) a_{m-\left(i-C_{r}\right)} b_{n-\left(j-D_{r}\right)}
\end{gathered}
$$

Define the generating function $G(y, z)$ as $\sum_{m} \sum_{n} \pi(m, n) y^{m} z^{n}$ for $|y| \leq 1$ and $|z| \leq 1$, then we can obtain

$$
\begin{gathered}
G(y, z)=\sum_{r=0}^{R-1} Q_{r} \sum_{i=0}^{C_{r}-1} \sum_{j=0}^{D_{r}-1} \pi(i, j) \sum_{m=0}^{\infty} a_{m} y^{m} \sum_{n=0}^{\infty} b_{n} z^{n}+ \\
\sum_{r=0}^{R-1} Q_{r} \sum_{i=0}^{C_{r}-1} \sum_{j=D_{r}}^{\infty} \pi(i, j) \sum_{m=0}^{\infty} a_{m} y^{m} \sum_{n=j-D_{r}}^{\infty} b_{n-\left(j-D_{r}\right)} z^{n}+ \\
\sum_{r=0}^{R-1} Q_{r} \sum_{i=C_{r}}^{\infty} \sum_{j=0}^{D_{r}-1} \pi(i, j) \sum_{m=i-C_{r}}^{\infty} a_{m-\left(i-C_{r}\right)} y^{m} \sum_{n=0}^{\infty} b_{n} z^{n}+ \\
\sum_{r=0}^{R-1} Q_{r} \sum_{i=C_{r}}^{\infty} \sum_{j=D_{r}}^{\infty} \pi(i, j) \sum_{m=i-C_{r}}^{\infty} a_{m-\left(i-C_{r}\right)} y^{m} \times \\
\sum_{n=j-D_{r}}^{\infty} b_{n-\left(j-D_{r}\right)} z^{n} .
\end{gathered}
$$

Let $A(y)=\sum_{m=0}^{\infty} a_{m} y^{m}$ and $B(z)=\sum_{n=0}^{\infty} b_{n} z^{n}$, we have

$$
\begin{gathered}
G(y, z)=\sum_{r=0}^{R-1} Q_{r} \sum_{i=0}^{C_{r}-1} \sum_{j=0}^{D_{r}-1} \pi(i, j) A(y) B(z)+ \\
\sum_{r=0}^{R-1} Q_{r} \sum_{i=0}^{C_{r}-1} \sum_{j=D_{r}}^{\infty} \pi(i, j) z^{j-D_{r}} A(y) B(z)+ \\
\sum_{r=0}^{R-1} Q_{r} \sum_{i=C_{r}}^{\infty} \sum_{j=0}^{D_{r}-1} \pi(i, j) y^{i-C_{r}} A(y) B(z)+ \\
\sum_{r=0}^{R-1} Q_{r} \sum_{i=C_{r}}^{\infty} \sum_{j=D_{r}}^{\infty} \pi(i, j) y^{i-C_{r}} z^{j-D_{r}} A(y) B(z),(23)
\end{gathered}
$$

Since $G(y, z)=\left(\sum_{i=0}^{C_{r}-1} \sum_{j=0}^{D_{r}-1}+\sum_{i=0}^{C_{r}-1} \sum_{j=D_{r}}^{\infty}+\sum_{i=C_{r}}^{\infty} \sum_{j=0}^{D_{r}-1}+\right.$ $\left.\sum_{\substack{i=C_{r} \\ \text { as }}}^{\infty} \sum_{j=D_{r}}^{\infty}\right) \pi(i, j) y^{i} z^{j}$, the last item in (23) can be rewritten

$$
\begin{aligned}
& \sum_{r=0}^{R-1} Q_{r} \sum_{i=C_{r}}^{\infty} \sum_{j=D_{r}}^{\infty} \pi(i, j) y^{i-C_{r}} z^{j-D_{r}} A(y) B(z)= \\
& \\
& \quad G(y, z) \sum_{r=0}^{R-1} Q_{r} y^{-C_{r}} z^{-D_{r}} A(y) B(z)- \\
& \sum_{r=0}^{R-1} Q_{r} \sum_{i=0}^{C_{r}-1} \sum_{j=0}^{D_{r}-1} \pi(i, j) y^{i-C_{r}} z^{j-D_{r}} A(y) B(z)- \\
& \sum_{r=0}^{R-1} Q_{r} \sum_{i=0}^{C_{r}-1} \sum_{j=D_{r}}^{\infty} \pi(i, j) y^{i-C_{r}} z^{j-D_{r}} A(y) B(z)- \\
& \sum_{r=0}^{R-1} Q_{r} \sum_{i=C_{r}}^{\infty} \sum_{j=0}^{D_{r}-1} \pi(i, j) y^{i-C_{r}} z^{j-D_{r}} A(y) B(z) .
\end{aligned}
$$

Substituting (24) in (23), we obtain

$$
\begin{aligned}
& G(y, z)\left[1-\sum_{r=0}^{R-1} Q_{r} y^{-C_{r}} z^{-D_{r}} A(y) B(z)\right]= \\
& \sum_{r=0}^{R-1} Q_{r} \sum_{i=0}^{C_{r}-1} \sum_{j=0}^{D_{r}-1} \pi(i, j)\left(1-y^{i-C_{r}} z^{j-D_{r}}\right) A(y) B(z)+ \\
& \sum_{r=0}^{R-1} Q_{r} \sum_{i=0}^{C_{r}-1} \sum_{j=D_{r}}^{\infty} \pi(i, j)\left(1-y^{i-C_{r}}\right) z^{j-D_{r}} A(y) B(z)+ \\
& \sum_{r=0}^{R-1} Q_{r} \sum_{i=C_{r}}^{\infty} \sum_{j=0}^{D_{r}-1} \pi(i, j) y^{i-C_{r}}\left(1-z^{j-D_{r}}\right) A(y) B(z) .
\end{aligned}
$$

Define the generating function $\Phi(y)=\sum_{m=0}^{\infty} \phi(m) y^{m}$ and $\Psi(z)=\sum_{n=0}^{\infty} \psi(n) z^{n}$. It is clear that $\Phi(1)=1=\Psi(1)$. Since 
$\pi(m, n)=\phi(m) \times \psi(n)$, we have the following equation:

$$
\Phi(y) \Psi(z)=\sum_{m=0}^{\infty} \sum_{n=0}^{\infty} \phi(m) \psi(n) y^{m} z^{n}=G(y, z) .
$$

Because of $\Phi(1)=1=\Psi(1)$ and $A(1)=\sum_{m} a_{m}=$ $1=\sum_{n} b_{n}=B(1)$, we can get the following equation after substituting $z=1$ in (26) and (25)

$$
\Phi(y)=\frac{A(y) \sum_{r=0}^{R-1} Q_{r} \sum_{i=0}^{C_{r}-1} \phi(i)\left(1-y^{i-C_{r}}\right)}{1-A(y) \sum_{r=0}^{R-1} Q_{r} y^{-C_{r}}} .
$$

Let $C=\max _{0 \leq r \leq R-1}\left\{C_{r}\right\}$. After the numerator and the denominator are both multiplied by $y^{C}, \Phi(y)$ can be computed by

$$
\Phi(y)=\frac{A(y) \sum_{r=0}^{R-1} Q_{r} \sum_{i=0}^{C_{r}-1} \phi(i)\left(y^{C}-y^{C+i-C_{r}}\right)}{y^{C}-A(y) \sum_{r=0}^{R-1} Q_{r} y^{C-C_{r}}} .
$$

Similarly, by substituting $y=1$ in (26) and (25), $\Psi(z)$ can also be obtained by

$\Psi(z)=\frac{B(z) \sum_{r=0}^{R-1} Q_{r} \sum_{j=0}^{D_{r}-1} \psi(j)\left(z^{D}-z^{D+j-D_{r}}\right)}{z^{D}-B(z) \sum_{r=0}^{R-1} Q_{r} z^{D-D_{r}}}$,

in which $D=\max _{0 \leq r \leq R-1}\left\{D_{r}\right\}$.

\section{REFERENCES}

[1] 3GPP TS 25.301 v6.1.0, "Radio Interface Protocol Architecture."

[2] 3GPP TS 25.302 v6.2.0, "Services provided by the physical layer."

[3] 3GPP TS 25.331 v6.4.0, "Radio Resource Control (RRC); protocol specification."

[4] 3GPP TS 25.212 v6.3.0, "Multiplexing and channel coding (FDD)."

[5] 3GPP TS 25.321 v6.3.0, "Medium Access Control (MAC) protocol specification."

[6] 3GPP TS 25.133 v6.8.0, "Requirements for support of radio resource management (FDD)."

[7] S.-T. Cheng, J.-L. Lin, and C.-Y. Wang, "TFCS selection algorithms in the MAC functionality of 3GPP," in Proc. International Computer Symposium 2002.

[8] K. Dimou and P. Godlewski, "MAC scheduling for uplink transmission in UMTS WCDMA," in Proc. IEEE Vehicular Technology Conference 2001, vol. 4, pp. 2625-2629.

[9] J. Pérez-Romero, O. Sallent, and R. Agustí, "Admission control for different UE-MAC algorithms in UTRA-UDD," in Proc. Third International Conference on $3 G$ Mobile Communication Technologies 2002 (Conf. Publ. No. 489), pp 256-260.

[10] O. Sallent, J. Pérez-Romero, R. Agustí, and F. Casadevall, "Provisioning multimedia wireless networks for better QoS: RRM strategies for $3 \mathrm{G} \mathrm{W}$ CDMA," IEEE Commun. Mag., vol. 41, pp. 100-106, Feb. 2003.

[11] O. Sallent, J. Pérez-Romero, F. J. Casadevall, and R. Agustí, "An emulator framework for a new radio resource management for QoS guaranteed services in W-CDMA systems," IEEE J. Select. Areas Commun., vol. 19, pp. 1893-1904, Oct. 2001.
[12] I. F. Akyldiz, D. A. Levine, and I. Joe, "A slotted CDMA protocol with BER scheduling for wireless multimedia networks," IEEE/ACM Trans. Networking, vol. 7, no. 2, pp. 146-158, Apr. 1999.

[13] T. S. Rappaport, Wireless Communications Principles and Practice. Prentice Hall PTR, 2002.

[14] H. Holma and A. Toskala, W-CDMA for UMTS. John Wiley and Sons, 2002.

[15] D. Gross and C. M. Harris, Fundamentals of Queueing Theory. John Wiley and Sons, 1998.

[16] J. H. Mathews and R. W. Howell, Complex Analysis for Mathematics and Engineering. Jones and Bartlett, 2000.

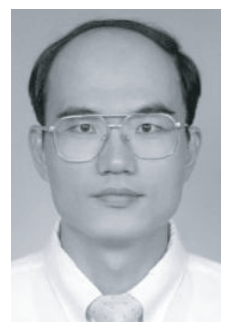

Sheng-Tzong Cheng received the B.S. (1985) and M.S. (1987) in Electrical Engineering from the National Taiwan University, Taipei, Taiwan. He received the M.S. (1993) and Ph.D. (1995) in Computer Science from the University of Maryland, College Park, MD. He was an Assistant Professor of Computer Science and Information Engineering at National Dong Hwa University, Hualien, Taiwan, in 1995, and became an Associate Professor in 1996. He is currently a Professor in the Department of Computer Science and Information Engineering, National Cheng Kung University, Tainan, Taiwan. His research interests are in design and performance analysis of mobile computing, wireless communications, multimedia, and real-time systems.

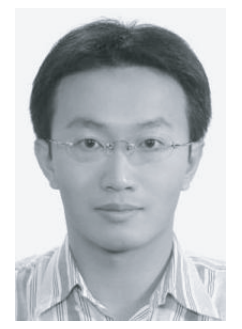

Chun-Yen Wang received the B.S. degree in mathematics from National Cheng Kung University, Tainan, Taiwan in 2001. He is currently working toward the Ph.D. degree in computer science and information engineering at National Cheng Kung University. His research interests include: wireless communications, mobile computing, quantum computation and quantum communications.

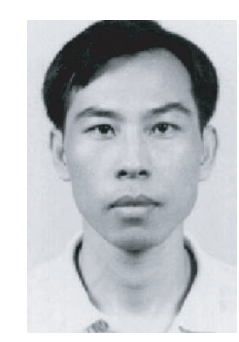

Ing-Ray Chen received the BS degree from the National Taiwan University, Taipei, Taiwan, and the $\mathrm{MS}$ and $\mathrm{PhD}$ degrees in computer science from the University of Houston. $\mathrm{He}$ is a professor in the Department of Computer Science at Virginia Tech. His research interests include mobile and pervasive computing, sensor systems, multimedia, distributed systems, real-time intelligent systems, and reliability and performance analysis. Dr. Chen has served on the program committee of numerous conferences, including as program chair for 29th IEEE Annual International Computer Software and Application Conference in 2005, 14th IEEE International Conference on Tools with Artificial Intelligence in 2002, and 3rd IEEE Symposium on Application-Specific Systems and Software Engineering Technology in 2000. Dr. Chen currently serves as an editor for Wireless Personal Communications, The Computer Journal, and the International Journal on Artificial Intelligence Tools. He is a member of the IEEE/CS and ACM. 